\title{
Desenvolvimento de uma Mesa X-Y Didática para Ensino Multidisciplinar em Engenharia Mecatrônica
}

DOI: 10.37702/2175-957X.COBENGE.2021.3615

Douglas Daniel Sampaio Santana - douglas.santana@usp.br

Universidade de São Paulo

Avenida Professor Mello de Morais 2231

05508-030 - Sao Paulo - SP

Newton Maruyama - maruyama@usp.br

Universidade de São Paulo

Av. Prof. Mello Moraes 2231

05508-030 - São Paulo - SP

Celso Massatoshi Furukawa - cmfuruka@usp.br

Universidade de Sao Paulo

Av Prof Mello Moraes 2231

05508-030 - Sao Paulo - SP

Resumo: Este artigo descreve um sistema didático em desenvolvimento para o ensino multidisciplinar em Engenharia Mecatrônica, envolvendo as áreas de Projeto Mecânico, Programação, Sistemas Embarcados, Sistemas de Tempo Real e Controle. Trata-se de um sistema cartesiano $X-Y$ acionada por servomotores $C C$ e controlado por microcontroladores de elevado poder computacional, o que permite a configuração por software de diferentes experimentos, conexão em rede e operação remota. Neste trabalho, foca-se na modelagem dinâmica detalhada e passo-a-passo do sistema, que poderá posteriormente ser trabalhado em disciplinas mais avançadas. A modelagem usa uma abordagem híbrida para controle de velocidade e posição e são apresentados resultados de simulações. Distúrbios de torque também foram incluídos e o sistema provou ser bastante robusto.

Palavras-chave: ensino multidisciplinar, Engenharia Mecatrônica, instrumentação virtual, modelagem e controle 


\section{Desenvolvimento de uma Mesa X-Y Didática para Ensino Multidisciplinar em Engenharia Mecatrônica}

\section{INTRODUÇÃO}

Projetos integrativos constituem uma moderna metodologia de formação profissional em diversas áreas, notadamente na Engenharia. Para a Engenharia Mecatrônica, são mais adequados projetos que integrem sistemas mecânicos, eletrônicos, computacionais e de controle, e que permitam uma abordagem multidisciplinar.

Apesar de, em um primeiro momento, parecer algo ambicioso, há atualmente uma grande disponibilidade de dispositivos e kits de boa qualidade e custos acessíveis. É uma área em rápido crescimento, impulsionada por projetos abertos de hardware e software de considerável complexidade, como impressoras 3D, drones, robôs móveis, etc. Por serem abertos, projetos desse tipo poderiam ser aplicados no ensino em Engenharia Mecatrônica. No entanto, a experiência dos autores é que sistemas completos apresentam grau de complexidade elevado, excesso de detalhes e, no caso dos projetos citados, baixa robustez para uso em cursos de graduação.

Procurando focar em projetos mais simples baseados em manipuladores cartesianos, observou-se uma deficiência principalmente na modelagem dinâmica, o que limita a aplicação desses sistemas no aprofundamento de aspectos teóricos. A maioria de projetos abertos dessa classe utilizam motores de passo, com operação em malha aberta. Além disso, por envolverem o controle conjunto de mais de um grau de liberdade e de outros parâmetros, os programas de firmware e de interface com o usuário são extensos e complexos (YOSHIKAWA, BRANCO) (GUETHI).

Buscou-se então desenvolver um sistema didático que possa ser utilizado em diferentes áreas da Engenharia Mecatrônica como: Projeto Mecânico; Programação; Eletrônica Embarcada; Sistemas de Tempo Real e Controle. Transversalmente, deseja-se integrar perfis de estudantes em diferentes estágios do curso, desde os primeiros semestres, passando pelos semestres finais, e avançando até o início da pós-graduação

Como principais requisitos, citam-se: o sistema deve ser ao mesmo tempo simples de entender e permitir abordagens teóricas mais avançadas, a ponto permitir o trabalho conjunto de estudantes de graduação e de mestrado em Engenharia; deve ser robusto; deve ter custo acessível e deve ser simples de ser operado.

Além disso, a disponibilidade atual de sistemas embarcáveis de notável poder computacional e capacidade de integração em rede permite propor um sistema que possa ser operado com pouca ou nenhuma intervenção humana. Com o uso de sensores e conversores $A / D$, a eletrônica embarcada é capaz de substituir custosos equipamentos de laboratório, como osciloscópios, fontes reguladas e geradores de função, além de eliminar a operação presencial para a geração de estímulos e captura de dados. Com isso, o sistema pode ser configurado pelos instrutores remotamente e usado pelos estudantes em experimentos à distância.

Optou-se então pelo desenvolvimento de um sistema de posicionamento cartesiano, acionado por servo-motores. Os principais elementos mecânicos e eletrônicos do sistema foram adquiridos, foi feita a montagem de um protótipo e foram feitos testes iniciais antes do início da pandemia. Infelizmente o afastamento forçado da equipe impediu a continuidade do projeto físico do sistema. Por esse motivo, optou-se por concentrar esforços na modelagem dinâmica do sistema e experimentos por simulação. 
O controle de velocidade e posicionamento cartesiano é uma técnica amplamente utilizada em diversos equipamentos, principalmente, em máquinas ferramentas. No entanto, efetuar o posicionamento com precisão não é algo trivial, pois esses sistemas estão sujeitos a uma série de perturbações internas e externas que inevitavelmente degradam o desempenho dos mesmos (PANDILOV, DUKOVISKI) (BREAZ et al). Neste sentido, torna-se necessário modelar uma planta e projetar um controlador que atue sobre o sistema de forma a garantir que o controle seja efetuado com precisão.

A partir de um sistema confiável de referência, vários experimentos didáticos podem ser desenvolvidos, desde exercícios de programação a técnicas avançadas de controle, sem que seja necessário modificar o sistema físico.

As seções a seguir descrevem a modelagem em detalhes. Os parâmetros usados no modelo refletem os que foram usados na montagem do protótipo.

\section{DEscriçÃo do Sistema}

A eletrônica embarcada do sistema usa uma plataforma $A R M$ para processamento de sinais, cálculo da malha de controle e interface de rede. A leitura e processamento dos sinais dos encoders é feita por uma placa arduino.

A Figura 1 mostra o sistema que se pretende controlar. O sistema possui duas malhas de controle que atuam sobre o servo-motor: uma malha de controle de velocidade (realimentada por um tacômetro) e outra malha de controle de posição (realimentada por um encoder óptico incremental).

Figura 1 - Representação da mesa coordenada no eixo x

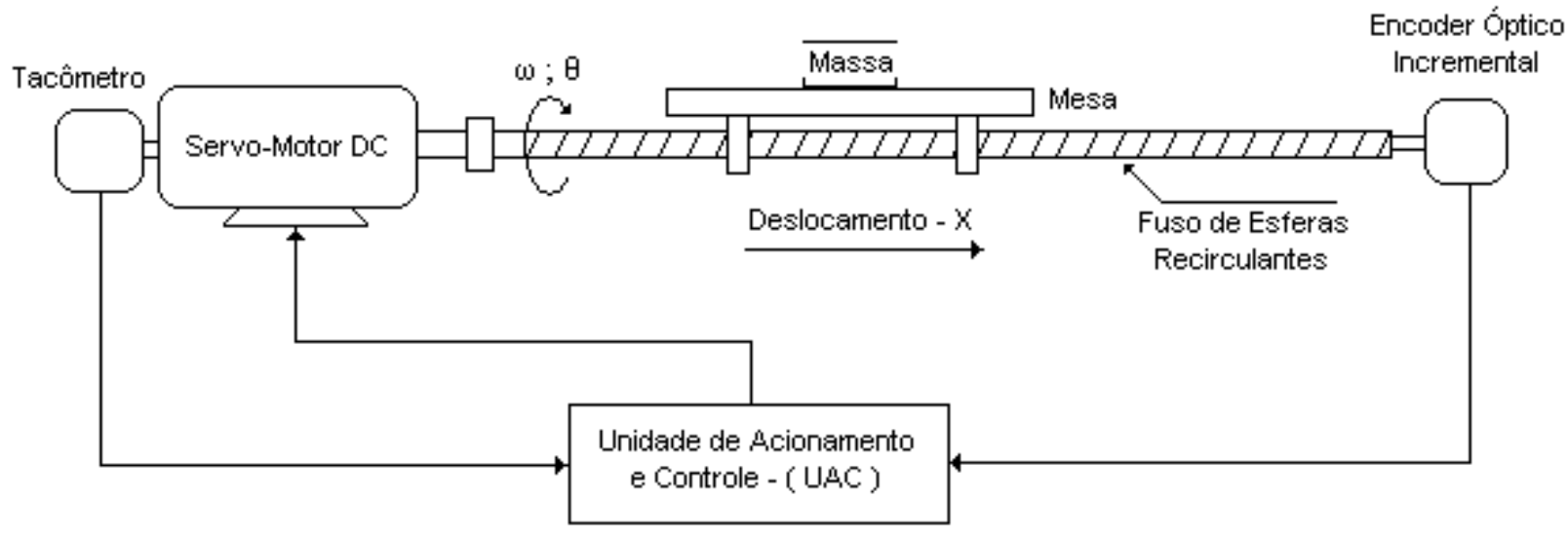

As principais metas desse trabalho são controlar a velocidade e o deslocamento da mesa coordenada dentro das especificações de projeto. A figura a seguir ilustra um eixo do sistema a ser controlado (neste exemplo o eixo x).

\section{Especificações de Projeto}

As especificações são feitas para o pior caso, ou seja, para o motor do eixo x, que transporta a mesa e todo o conjunto do eixo y. Também são apresentados os critérios de desempenho desejados para as malhas de velocidade e posição. 
Tabela 1 - Especificações de desempenho das malhas de velocidade e de posição

\begin{tabular}{l|c|l}
\hline Malha de Velocidade & Símb. & Valor \\
\hline Velocidade da mesa & $V_{L}$ & $0,2 \mathrm{~m} / \mathrm{s}$ \\
\hline Tempo de subida & $t_{r}$ & $15 \mathrm{~ms}$ \\
\hline Tempo de pico & $t_{p}$ & $20 \mathrm{~ms}$ \\
\hline Tempo de & $t_{s}$ & 0,1 \\
assentamento (2\%) & $M_{p}$ & $5 \%$ \\
\hline Massa do fuso & \multicolumn{2}{|l}{}
\end{tabular}

\begin{tabular}{l|c|l}
\hline Malha de Posição & Símb. & Valor \\
\hline Tempo de subida & $t_{r}$ & $60 \mathrm{~ms}$ \\
\hline Tempo de pico & $t_{p}$ & $80 \mathrm{~ms}$ \\
\hline Tempo de assentamento (2\%) & $t_{s}$ & $0,15 \mathrm{~s}$ \\
\hline Máximo sobre-sinal & $M_{p}$ & $1 \%$ \\
\hline & & \\
\hline
\end{tabular}

Descreve-se a seguir as especificações do conjunto mesa, fuso e guias lineares usados na construção do protótipo.

Tabela 2 - Especificações do conjunto mesa, fuso e guias

\begin{tabular}{l|c|l}
\hline Parâmetro & Símb. & Valor \\
\hline Massa da peça & $m_{p}$ & $50 \mathrm{~kg}$ \\
\hline Massa da mesa & $m_{m}$ & $20 \mathrm{~kg}$ \\
\hline Comprimento do fuso & $l_{f}$ & $800 \mathrm{~mm}$ \\
\hline Diâmetro do fuso & $d_{f}$ & $15 \mathrm{~mm}$ \\
\hline Massa do fuso & $m_{f}$ & $1,5 \mathrm{~kg}$ \\
\hline
\end{tabular}

\begin{tabular}{l|c|l}
\hline Parâmetro & Símb. & Valor \\
\hline Passo do fuso & $p_{f}$ & $5 \mathrm{~mm} / \mathrm{volta}$ \\
\hline Coef. friç̧ão dos rolamentos & $\mu_{b}$ & 0,005 \\
\hline Comprimento da guia linear & $l_{g}$ & $800 \mathrm{~mm}$ \\
\hline Coeficiente de fricção das guias & $\mu_{g}$ & 0,05 \\
\hline Força de pré-carga no fuso & $F_{P}$ & $2500 \mathrm{~N}$ \\
\hline
\end{tabular}

\subsection{Análise dos carregamentos e escolha do servomotor}

Para escolher um servo-motor adequado, torna-se necessário calcular os carregamentos estáticos e dinâmicos do conjunto motor-fuso (ROTHBART, BROWN). Os torques refletidos no eixo do fuso devido à fricção nas guias, pré-carga e total são:

$$
\begin{aligned}
& T_{g}=\frac{p_{f}}{2 \pi} \mu_{g}\left(m_{m}+m_{p}\right) g=\frac{0,005}{2 \pi} 0,05(20+50) 9,82=0,0274[\mathrm{Nm}] \\
& T_{p f}=\mu_{b} \frac{d_{f}}{2} F_{p}=0,005 \frac{0,015}{2} 2500=0,0937[\mathrm{Nm}] \\
& T_{e}=T_{g}+T_{p f}=(27,4+93,7) \times 10^{-3}=121,1 \times 10^{-3}[\mathrm{Nm}]
\end{aligned}
$$

Os momentos de inércia refletido no eixo do fuso devido às massas da mesa, da peça, do próprio fuso e total, são:

$$
\begin{gathered}
J_{p m}=\left(m_{m}+m_{p}\right)\left(\frac{p_{f}}{2 \pi}\right)^{2}=(20+50)\left(\frac{0,005}{2 \pi}\right)^{2}=44,33 \times 10^{-6}\left[\mathrm{Kgm}^{2}\right] \\
J_{f}=\frac{1}{2} m_{f}\left(\frac{d_{f}}{2}\right)^{2}=\frac{1,5}{2}\left(\frac{0,015}{2}\right)^{2}=42,2 \times 10^{-6}\left[\mathrm{Kgm}^{2}\right] \\
J_{t}=J_{p m}+J_{f}+J_{r}=44,3 \times 10^{-6}+42,2 \times 10^{-6}+20 \times 10^{-6}=106,5 \times 10^{-6}\left[\mathrm{Kgm}^{2}\right] \\
a_{l}=\frac{V_{l}}{t_{r}}=\frac{0,2}{0,01}=20\left[\mathrm{~m} / \mathrm{s}^{2}\right] \\
\frac{d \omega}{d t}=\frac{a_{l}}{\left(p_{f} / 2 \pi\right)}=\frac{2 \pi a_{l}}{p_{f}}=\frac{2 \pi 20}{0,005} \simeq 25133\left[\mathrm{rad} / \mathrm{s}^{2}\right] \\
T_{d}=J_{t} \frac{d \omega}{d t}+B \omega+T_{e}=106,5 \times 10^{-6} \times 25133+121,1 \times 10^{-3}=2,72[\mathrm{Nm}]
\end{gathered}
$$

Para estimar a aceleração linear, a aceleração angular e o torque dinâmico total, a equação 3 considera que o coeficiente de atrito viscoso $(B)$ é desprezível. 
O motor a ser escolhido deve ser capaz de suportar um torque dinâmico de 2,72 Nm num período de $0,01 \mathrm{~s}$. A potência do motor pode ser obtida através do produto do torque pela velocidade angular (ALTINTAS):

$$
P_{\text {motor }}=T \omega=T_{d} \int_{0}^{t_{r}} \frac{d \omega}{d t} d t=2,72 \int_{0}^{0,01} 25133 d t=683,6[\mathrm{~W}] .
$$

A seguir são descritas as especificações do servo-motor CC escolhido de acordo com as necessidades acima mencionadas. Os dados foram retirados do catalogo do fabricante (servo-motor CC de 800W da "Applied Motion"). Os dispositivos de realimentação são partes integrantes do servo-motor escolhido.

Tabela 3 - Especificações do servo-motor escolhido

\begin{tabular}{l|c|l}
\hline Parâmetro & Símb. & Valor \\
\hline Inércia rotor & $J_{r}$ & $20 \times 10^{-6} \mathrm{Kgm}^{2}$ \\
\hline Coef. de atrito viscoso & $B$ & $0,0001 \mathrm{Nms}$ \\
\hline Resistência de armadura & $R_{a}$ & $2,4 \Omega$ \\
\hline Indutância de armadura & $L_{a}$ & $0,0275 \mathrm{H}$ \\
\hline
\end{tabular}

\begin{tabular}{l|c|l}
\hline Parâmetro & Símb. & Valor \\
\hline Constante de torque & $K_{\tau}$ & $0,046 \mathrm{Nm} / \mathrm{A}$ \\
\hline Constante elétrica & $K_{b}$ & $0,044 \mathrm{Vs} / \mathrm{rad}$ \\
\hline $\begin{array}{l}\text { Constante do } \\
\text { tacômetro }\end{array}$ & $H_{g}$ & $0,01 \mathrm{~V} / \mathrm{rpm}$ \\
\hline Constante do encoder & $K_{e}$ & $1000 \mathrm{ppr}$ \\
\hline
\end{tabular}

4

MODELAGEM DO SISTEMA

A Figura 2 apresenta a estrutura de controle empregada no controle de velocidade e posicionamento dos servo-motores dos eixos $x$ e y da mesa coordenada.

Figura 2 - Diagrama de blocos da estrutura de controle proposta

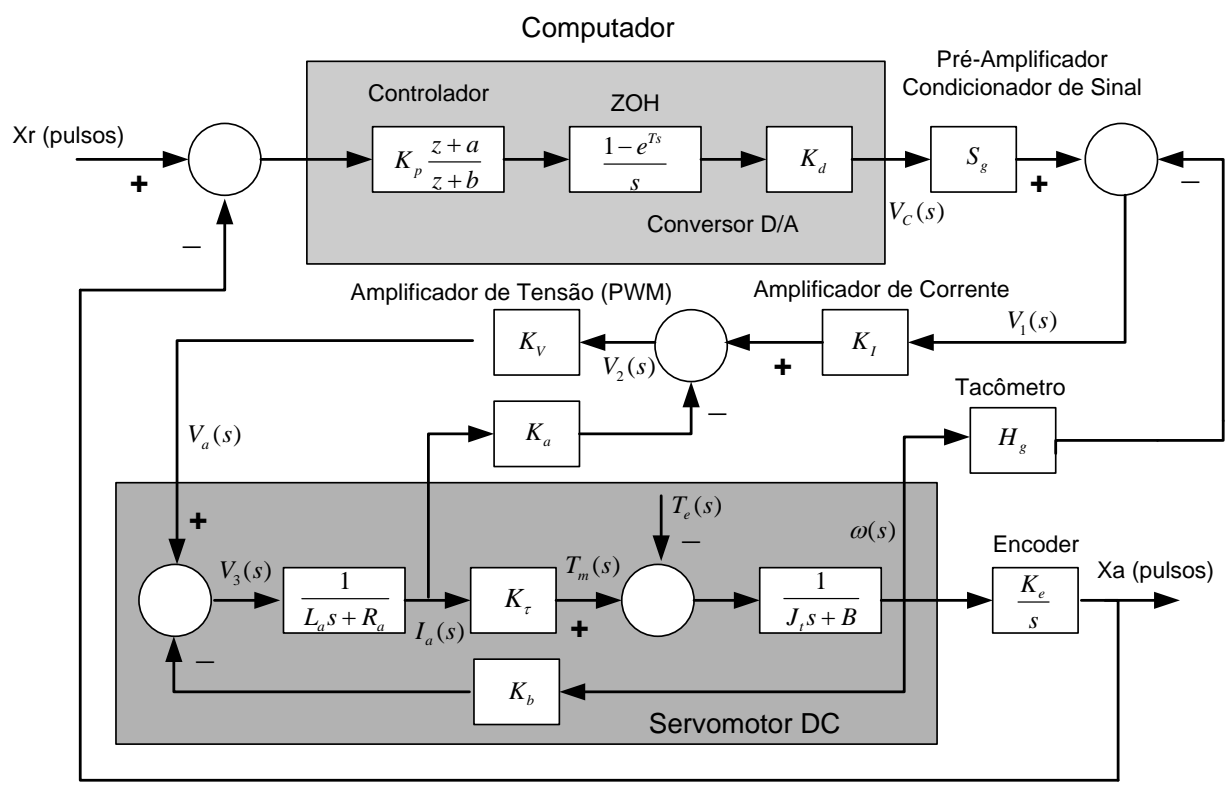

O sistema de realimentação proposto utiliza dois tipos de sensores: um para realimentação de velocidade (tacômetro) e outro para realimentação de posição (encoder incremental). A tensão de saída de um tacômetro operando na região linear é dada pelo produto da constante do tacômetro $\left(H_{g}\right)$ pela velocidade angular $(\omega)$. Um encoder incremental fornece uma certa quantidade de pulsos por unidade de deslocamento (angular ou linear). O sinal do encoder é aplicado num contador "up-down", portanto com o passar do tempo, tem-se uma somatória dos pulsos, ou seja, uma ação integral. 


$$
\begin{aligned}
& V_{\text {taco }}(t)=\omega(t) H_{\text {taco }}=\frac{V_{\text {taco }}(\mathrm{s})}{\omega(\mathrm{s})}=H_{g}(\mathrm{~s})[\mathrm{V} / \mathrm{rad} / \mathrm{s}] \\
& \frac{p_{e}}{2 \pi}=\frac{K_{e}}{\mathrm{~s}}[\text { Pulsos } / \mathrm{rad}]
\end{aligned}
$$

A partir do diagrama de blocos da Figura 2, podem-se estabelecer três variáveis auxiliares, $\bigvee_{1}, V_{2}$ e $V_{3}$, que são expressas por:

$$
\begin{aligned}
& V_{1}(s)=S_{g} V_{c}(s)-H_{g} \omega(s) \\
& V_{2}(s)=K_{I} S_{g} V_{c}(s)-K_{I} H_{g} \omega(s)-K_{a} I_{a}(s) \\
& V_{3}(s)=K_{V} K_{I} S_{g} V_{c}(s)-\left(K_{V} K_{I} H_{g}+K_{b}\right) \omega(s)-K_{V} K_{a} I_{a}(s)
\end{aligned}
$$

A corrente de armadura também pode ser expressa por:

$$
I_{a}(s)=\frac{V_{3}(s)}{L_{a} s+R_{a}}=\frac{K_{V} K_{I} S_{g}}{L_{a} s+R_{a}+K_{V} K_{a}} V_{C}(s)-\frac{K_{V} K_{I} H_{g}+K_{b}}{L_{a} s+R_{a}+K_{V} K_{a}} \omega(s)
$$

A velocidade angular do motor pode ser expressa por:

$$
\omega(s)=\frac{T_{m}(s)-T_{s}(s)}{J_{t} s+B}=\frac{K_{t}}{J_{t} s+B} I_{a}(s)-\frac{1}{J_{t} s+B} T_{s}(s)
$$

O diagrama de blocos da Figura 2 permite escrever a velocidade angular como:

$$
\begin{gathered}
\omega(s)=\frac{K_{1} V_{C}(s)-\frac{\left(\frac{1}{J_{t}}\right)\left(s+\frac{R_{a}+K_{V} K_{a}}{L_{a}}\right)}{s^{2}+K_{2} s+K_{2} s+K_{3}} T_{s}(s)}{K_{1}=\frac{K_{t} S_{g} K_{I} K_{V}}{L_{a} J_{t}} ; K_{2}=\frac{B}{J_{t}}+\frac{R_{a}+K_{V} K_{a}}{L_{a}} ; K_{3}=\frac{B\left(R_{a}+K_{V} K_{a}\right)+K_{t}\left(K_{b}+H_{g} T_{g} K_{V} K_{I}\right)}{J_{t} L_{a}}}
\end{gathered}
$$

O controle de velocidade deve ser projetado de forma a obter um tempo de subida rápido com sobressinal muito pequeno (ZHOU). Portanto, vamos analisar a componente da equação 8 , que relaciona a velocidade $\omega(s)$ com a tensão de entrada $V_{C}(s)$ do condicionador de sinal (pré-amplificador):

$$
\begin{aligned}
& \frac{\omega(s)}{V_{C}(s)}=\frac{b_{0} \omega_{n}{ }^{2}}{s^{2}+2 \zeta \omega_{n} s+\omega_{n}{ }^{2}}=\frac{K_{1}}{s^{2}+K_{2} s+K_{3}} \\
& K_{2}=2 \zeta \omega_{n} \quad ; \quad K_{3}=\omega_{n}{ }^{2}
\end{aligned}
$$

Em servo drives para sistemas de posicionamento, é comum utilizar o tempo de pico para a malha de velocidade por volta de $10 \mathrm{~ms}$ e sobressinal em torno de $5 \%$ (ALTINTAS) (ZHAO). Portanto, o coeficiente de amortecimento $\zeta$ e a frequência natural $\omega_{n}$ são obtidos por:

$$
\begin{aligned}
& \zeta=\sqrt{\frac{\left(\ln \left(M_{P}\right)\right)^{2}}{\left(\ln \left(M_{P}\right)\right)^{2}+\pi^{2}}}=\sqrt{\frac{(\ln (0,05))^{2}}{(\ln (0,05))^{2}+\pi^{2}}}=0,83 \\
& \omega_{n}=\frac{\pi}{t_{p} \sqrt{1-\zeta^{2}}}=\frac{\pi}{0.01 \sqrt{1-0,826^{2}}}=452,7 \quad[\mathrm{rad} / \mathrm{s}]
\end{aligned}
$$


Substituindo a equação 10 na equação 9, determinam-se os valores de $K_{2}$ e $K_{3}$ :

$$
\begin{aligned}
& K_{2}=2 \zeta \omega_{n}=2 \times 0,83 \times 452,7=751,5 \\
& K_{3}=\omega_{n}{ }^{2}=\frac{B\left(R_{a}+K_{V} K_{a}\right)+K_{t}\left(K_{b}+H_{g} K_{V} K_{I}\right)}{J_{t} L_{a}}=452,7^{2}
\end{aligned}
$$

A equação 8 também permite determinar o produto $K_{V} K_{a}$ :

$$
K_{V} K_{a}=\frac{\left(K_{2} J_{t}-B\right) L_{a}}{J_{t}}-R_{a}=\frac{\left(751,5 \times 106,5 \times 10^{-6}-10^{-4}\right) 27,5 \times 10^{-3}}{106,5 \times 10^{-6}}-2,4=18,24
$$

Adotando um ganho de tensão $K_{V}=10$, os valores para os amplificadores $K_{I}, K_{1}$ podem ser determinados. Em seguida determina-se $S_{g}$ :

$$
\begin{gathered}
K_{I}=\frac{\omega_{n}^{2} J_{t} L_{a}-B\left(R_{a}+K_{V} K_{a}\right)-K_{t} K_{b}}{K_{t} H_{g} T_{g} K_{V}}=0,4072 \\
K_{1}=\frac{K_{t} S_{g} K_{I} K_{V}}{L_{a} J_{t}}=\frac{0,08 \times S_{g} \times 0,4072 \times 10}{0,00275 \times 71,6959 \times 10^{-6}}=1652136,4 . S_{g} \\
\frac{\omega(s)}{V_{C}(s)}=\frac{K_{1}}{s^{2}+K_{2} s+K_{3}}=\frac{\omega_{n}{ }^{2}}{s^{2}+2 \zeta \omega_{n} s+\omega_{n}{ }^{2}}=\frac{1652136,43 . S_{g}}{s^{2}+651,9 s+204937,3} \\
S_{g}=\frac{452,7^{2}}{1652136,43} \simeq 0,125
\end{gathered}
$$

Conforme a Figura 2, a tensão aplicada na armadura pode ser expressa por:

$$
V_{a}=K_{V}\left[K_{I}\left(S_{g} V_{c}-H_{g} \omega\right)-K_{a} I_{a}\right]=R_{a} I_{a}+L_{a} \frac{d I_{a}}{d t}+K_{b} \omega
$$

Igualando os torques elétricos e mecânicos e aplicando a transformada de Laplace inversa na função de transferência do contador "up-down" da Figura 2 tem-se:

$$
\begin{aligned}
& R_{a} I_{a}+L_{a} \frac{d I_{a}}{d t}+K_{b} \omega=K_{V}\left[K_{I}\left(S_{g} V_{c}-H_{g} \omega\right)-K_{a} I_{a}\right] \\
& \frac{d I_{a}}{d t}=-\frac{K_{V} K_{a}-R_{a}}{L_{a}} I_{a}-\frac{K_{V} K_{I} H_{g}-K_{b}}{L_{a}} \omega+\frac{K_{V} K_{I} S_{g}}{L_{a}} V_{c} \\
& J_{t} \frac{d \omega}{d t}+B \omega+T_{e}=K_{\tau} \cdot I_{a} \rightarrow \frac{d \omega}{d t}=\frac{K_{\tau}}{J_{t}} I_{a}-\frac{B}{J_{t}} \omega-\frac{1}{J_{t}} T_{e} \\
& X_{a}(s)=\frac{K_{e}}{s} \omega(s) \rightarrow s X_{a}(s)=K_{e} \omega(s) \rightarrow \frac{d X_{a}}{d t}=K_{e} \omega
\end{aligned}
$$

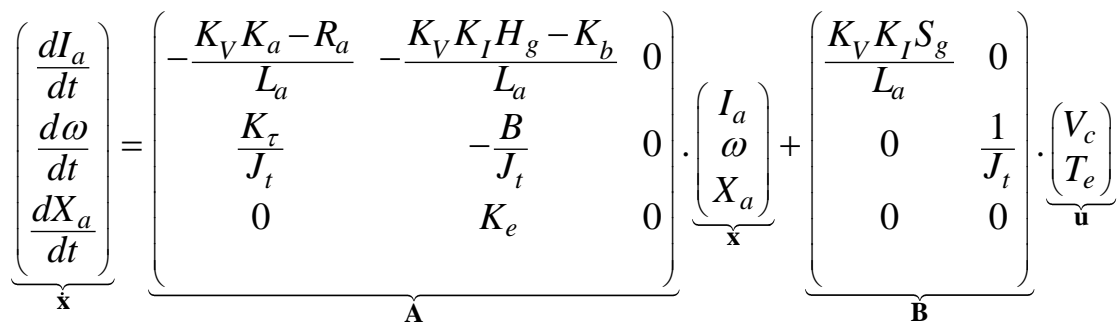


A equação 16 pode ser discretizada num intervalo de tempo $\mathrm{T}$ por $\mathbf{x}[k+1]=\mathbf{A}_{\mathbf{d}}(T) \mathbf{x}[k]+\mathbf{B}_{\mathbf{d}}(T) \mathbf{u}[k]($ OGATA) (VU, SFANDIARI), onde:

$$
\mathbf{A}_{\mathbf{d}}(T)=e^{\mathbf{A} T}=\left(\begin{array}{lll}
\phi_{11} & \phi_{12} & \phi_{13} \\
\phi_{21} & \phi_{22} & \phi_{23} \\
\phi_{31} & \phi_{32} & \phi_{33}
\end{array}\right) \quad \mathbf{B}_{\mathbf{d}}(T)=\left(\int_{0}^{T} e^{\mathbf{A} T} d t\right) \mathbf{B}=\left(\begin{array}{ll}
h_{11} & h_{12} \\
h_{21} & h_{22} \\
h_{31} & h_{32}
\end{array}\right)
$$

A parte digital do controle de posicionamento é composta pelo filtro digital (controlador) e pelo ganho $\mathrm{K}_{d}$ do conversor D/A. A partir da Figura 2 o sinal para comando de velocidade pode ser expresso no domínio $z$, por:

$$
\begin{gathered}
V_{c}[k]=K_{p} \frac{z+a}{z+b} K_{d}\left(X_{r}[k]-X_{a}[k]\right) \\
V_{c}[k]=K_{p} K_{d}\left(X_{r}[k]-X_{a}[k]\right)+V_{d}[k]=-K_{p} K_{d} X_{a}[k]+K_{p} K_{d} X_{r}[k]+V_{d}[k] \\
V_{d}[k]=K_{p} K_{d} \frac{a-b}{z+b}\left(X_{r}[k]-X_{a}[k]\right)
\end{gathered}
$$

A nova variável $V_{d}$ é uma tensão digital que descreve o estado interno do filtro, e assim sendo, será tratada como uma quarta variável de estado. A tensão $V_{c}$ é a saída do conversor D/A e interessa ser conhecida, portanto, será tratada como uma variável de saída (medida). Depois de discretizar a planta utilizando o modelo descrito pela equação $18 \mathrm{e}$ após algumas manipulações algébricas obtêm-se as seguintes equações com quatro estados discretos:

$$
\begin{aligned}
& V_{d}[k+1]=-b V_{d}[k]-K_{p} K_{d}(a-b) X_{a}[k]+K_{p} K_{d}(a-b) X_{r}[k] \\
& I_{a}[k+1]=h_{11} V_{d}[k]+\phi_{11} I_{a}[k]+\phi_{12} \omega[k]+\left(\phi_{13}-h_{11} K_{p} K_{d}\right) X_{a}[k]+h_{11} K_{p} K_{d} X_{r}[k]+h_{12} T_{e}[k] \\
& \omega[k+1]=h_{21} V_{d}[k]+\phi_{21} I_{a}[k]+\phi_{22} \omega[k]+\left(\phi_{23}-h_{21} K_{p} K_{d}\right) X_{a}[k]+h_{21} K_{p} K_{d} X_{r}[k]+h_{22} T_{e}[k] \\
& X_{a}[k+1]=h_{31} V_{d}[k]+\phi_{31} I_{a}[k]+\phi_{32} \omega[k]+\left(\phi_{33}-h_{31} K_{p} K_{d}\right) X_{a}[k]+h_{31} K_{p} K_{d} X_{r}[k]+h_{32} T_{e}[k]
\end{aligned}
$$

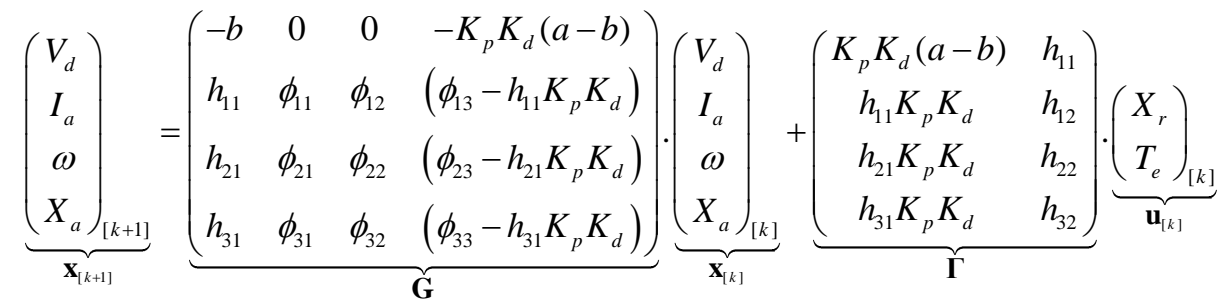

$$
\begin{aligned}
& \underbrace{\left(\begin{array}{c}
V_{c} \\
I_{a} \\
\omega \\
X_{a}
\end{array}\right)_{[k]}}_{\mathbf{y}_{[k]}}=\underbrace{\left(\begin{array}{cccc}
1 & 0 & 0 & -K_{p} K_{d} \\
0 & 1 & 0 & 0 \\
0 & 0 & 1 & 0 \\
0 & 0 & 0 & 1
\end{array}\right)}_{\mathbf{C}} \cdot \underbrace{\left(\begin{array}{c}
V_{d} \\
I_{a} \\
\omega \\
X_{a}
\end{array}\right)_{[k]}}_{\mathbf{x}_{[k]}}+\underbrace{\left(\begin{array}{ccc}
K_{p} K_{d} & 0 \\
0 & 0 \\
0 & 0 \\
0 & 0
\end{array}\right)}_{\mathbf{D}} \cdot \underbrace{\left(\begin{array}{c}
X_{r} \\
T_{e}
\end{array}\right)_{[k]}}_{\mathbf{u}_{[k]}}
\end{aligned}
$$

Com o auxílio do MATLAB®, obteve-se o seguinte compensador contínuo:

$$
C(s)=K_{p c} \frac{s+a}{s+b}=16,2 \frac{s+1265,8}{s+759,3}
$$


A frequência de amostragem foi escolhida para ser igual a 15 vezes a frequência natural do sistema, ou seja, $15 \omega_{n}=15 \times 452,7[\mathrm{rad} / \mathrm{s}] \simeq 1080[\mathrm{~Hz}]$. Assim o período de amostragem fica sendo: $1 / 1080 \simeq 0,001[s]$. Aplicando a transformação casada $z=e^{s T}$ temos os seguintes polos: $z=e^{-1265,8 \times 0,001}=0,2820$ e $z=e^{-759,3 \times 0,001}=0,4640$. Para casar os filtros em baixa frequência, tem-se que quando $s \rightarrow 0, z \rightarrow 1$. Portanto, o compensador (filtro) digital fica sendo (OGATA):

$$
\begin{gathered}
16,2 \frac{0+1265,8}{0+759,3}=K_{p d} \frac{1-0.2820}{1-0.4680} \Rightarrow K_{p d}=20 \\
D(z)=K_{p d} \frac{z+a}{z+b}=20 \frac{z-0.2820}{z-0.4680}
\end{gathered}
$$

Substituindo-se os valores de $K_{p}, K_{d}, a, b, \phi_{i j} e h_{i j}$ na equação 20 chegou-se as seguintes matrizes:

$$
\begin{aligned}
& \mathbf{G}(T)=\left(\begin{array}{cccc}
0.4680 & 0 & 0 & -0.0727 \\
0.1642 & 0.5604 & -0.1567 & -0.0641 \\
0.0399 & 0.3394 & 0.9543 & -0.0156 \\
0.0011 & 0.0148 & 0.0782 & 0.9996
\end{array}\right) \quad \boldsymbol{\Gamma}(T)=\left(\begin{array}{cc}
0.0727 & 0 \\
0.0641 & 0.8145 \\
0.0156 & -9.3155 \\
0.0004 & -0.3736
\end{array}\right) \\
& \mathbf{C}(T)=\left(\begin{array}{cccc}
1 & 0 & 0 & -0.3906 \\
0 & 1 & 0 & 0 \\
0 & 0 & 1 & 0 \\
0 & 0 & 0 & 1
\end{array}\right) \quad \mathbf{D}(T)=\left(\begin{array}{cc}
0.3906 & 0 \\
0 & 0 \\
0 & 0 \\
0 & 0
\end{array}\right)
\end{aligned}
$$

\section{$5 \quad$ RESULTADOS E DISCUSSÕES}

A seguir são mostrados os resultados obtidos através da simulação em ambiente MATLAB $®$. O sistema foi simulado com entrada do tipo degrau "com e sem" perturbação de torque externo. Também foram feitas simulações com perfis de entrada do tipo rampa e senoidal. Realizaram-se interpolações circulares "com e sem" perturbação de torque.

Figura 3 - (a) deslocamento $\mathrm{x}$ corrente e $(\mathrm{b})$ deslocamento $\mathrm{x}$ velocidade

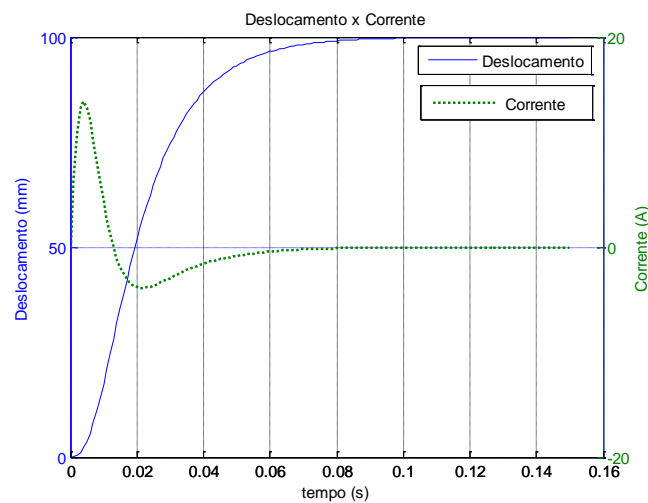

(a)

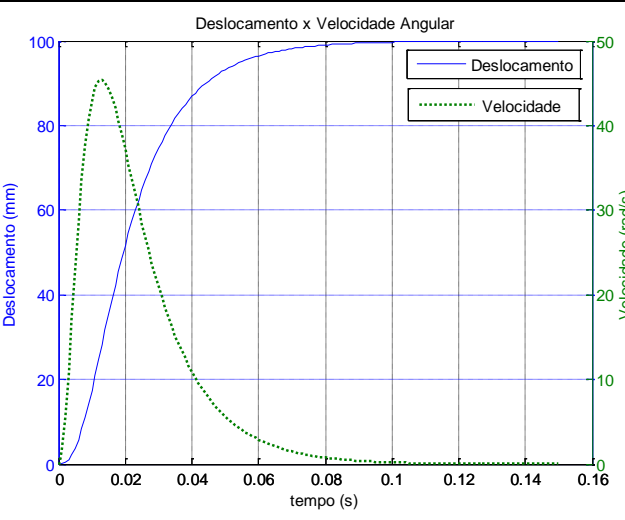

(b) 
A Figura 3 mostra que o tempo de assentamento $\left(t_{s}\right)$ é de aproximadamente $0,1 \mathrm{~s}$, e que não ocorre sobressinal, o que está de acordo com as especificações de desempenho propostas. Esta figura também ilustra que o comportamento da corrente e da velocidade são coerentes, pois no instante em que o deslocamento atinge o sinal de comando tanto a corrente quanto a velocidade são nulos. Nota-se ainda que em alguns trechos a corrente assume valores negativos, indicando que o torque do motor devido a inércia é maior que o torque elétrico $T_{m}(s)=K_{\tau} \cdot I_{a}(s)$.

As figuras a seguir ilustram a resposta do sistema quando se aplica uma entrada do tipo rampa (Figura 4a e Figura 4b) e uma entrada do tipo senoidal (Figura 5a e Figura 5b). Nestas simulações não foram inseridas perturbações de torque, e verifica-se que o sistema acompanha o sinal de referência satisfatoriamente. Verifica-se ainda que os perfis de velocidade apresentam valores compatíveis com o esperado, pois, ao analisar visualmente as curvas de "deslocamento x velocidade", observa-se que as mesmas são as derivadas dos deslocamentos (vide Figura $4 b$ e Figura $5 b$ ).

Figura 4 - Resposta para entrada do tipo rampa

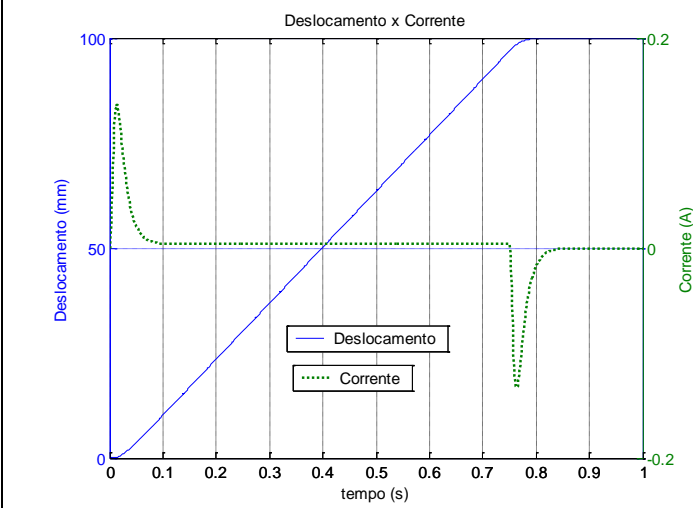

(a)

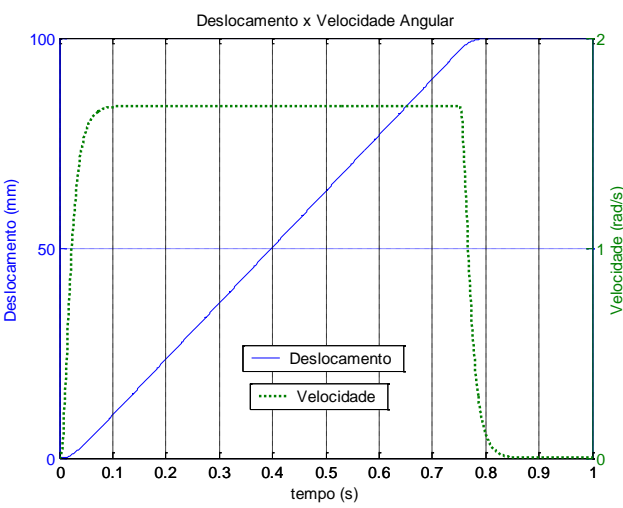

(b)

Figura 5 - Resposta para entrada do tipo senoidal

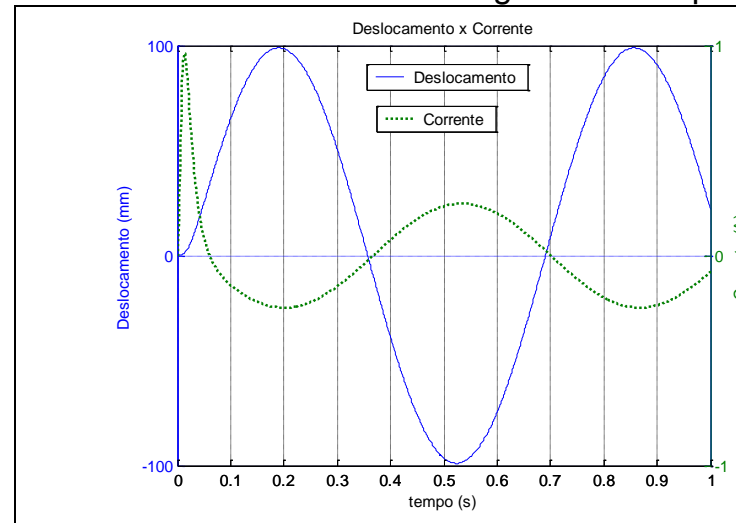

(a)

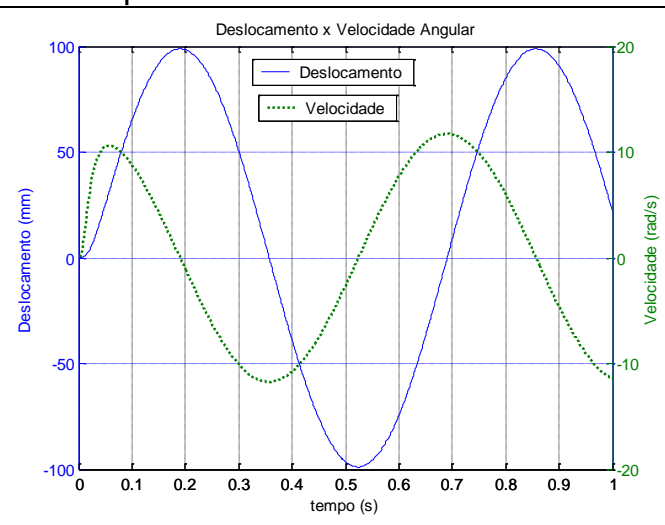

(b)

A seguir são apresentados os resultados para operações de interpolações circulares. A Figura 6 apresenta o resultado da simulação sem perturbação de torque, enquanto que a Figura 8 apresenta o resultado da interpolação com perturbação de torque igual a $20 \%$. Dado que nas literaturas pesquisadas, não se encontrou nenhuma informação a respeito de índices de desempenho para este tipo de operação (interpolação), adotou-se como índice de desempenho a medida do erro quadrático: (vide Figura 7 e Figura 9). 
Figura 6 - Interpolação sem perturbação no torque de entrada

(a) interpolação completa e (b) trecho ampliado para verificação do erro

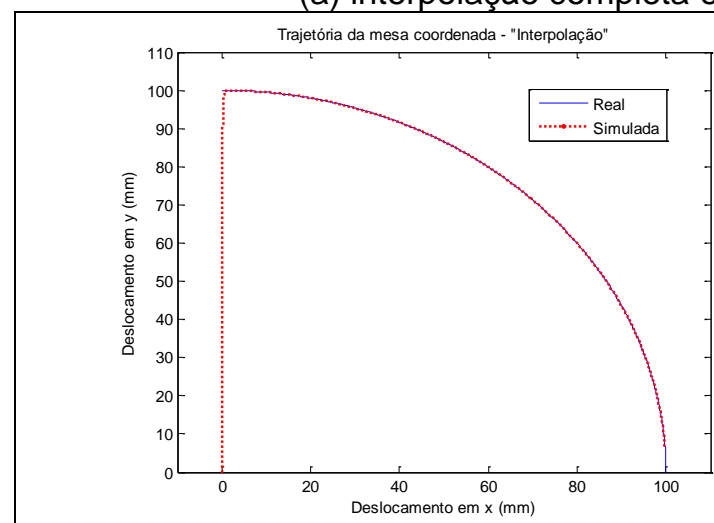

(a)

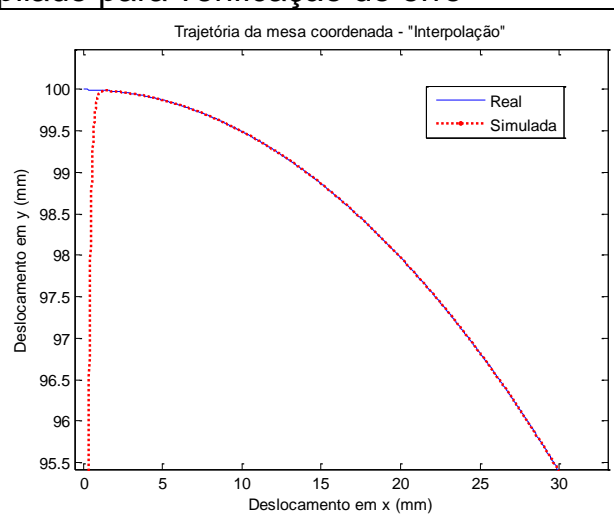

(b)

Figura 7 - Erro quadrático sem perturbação no torque de entrada

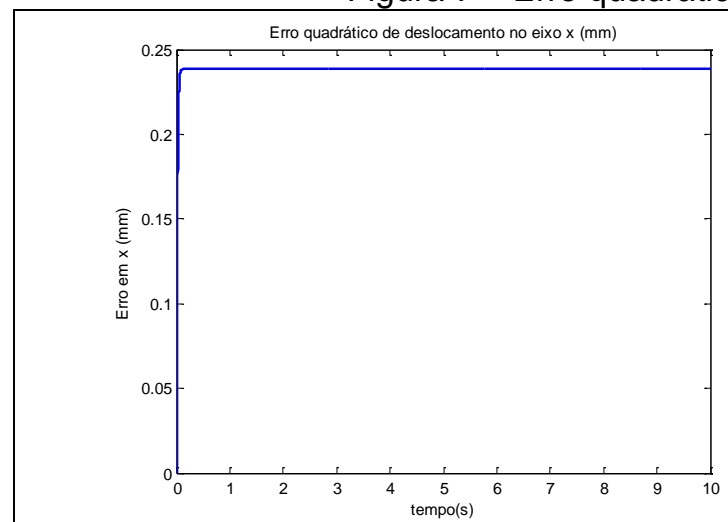

(a)

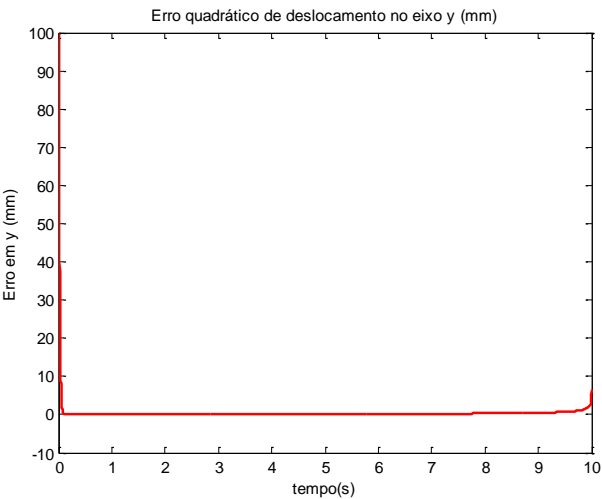

(b)

A Figura 7 mostra que assim que o sistema se assenta os erros de posicionamento em x e y, são de aproximadamente $0,23 \mathrm{~mm}$, ou seja, o erro do raio é de aproximadamente $e_{\text {raio }}=\sqrt{e_{x}^{2}+e_{y}^{2}}=\sqrt{0,23^{2}+0,23^{2}}=0,23 \sqrt{2}=0,33 \mathrm{~mm}$. Assim, para um raio de interpolação de $100 \mathrm{~mm}$ tem-se um erro de $0,33 \%$. Como a especificação de desempenho é de $1 \%$, admitese que o sistema esta operando adequadamente.

Figura 8 - Interpolação com perturbação no torque de entrada (a) interpolação completa e (b) trecho ampliado para verificação do erro

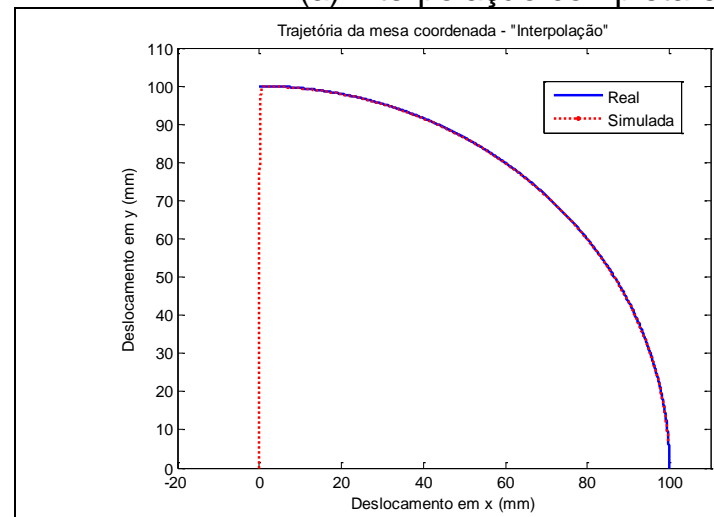

(a)

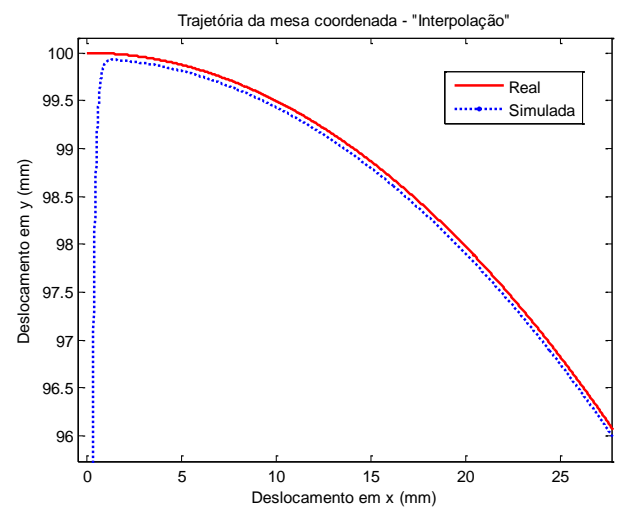

(b) 
Figura 9 - Erro quadrático com perturbação de $20 \%$ no torque de entrada

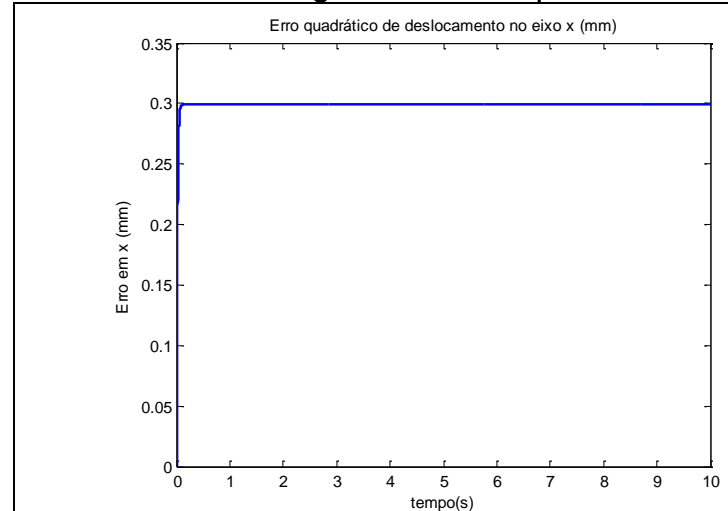

(a)

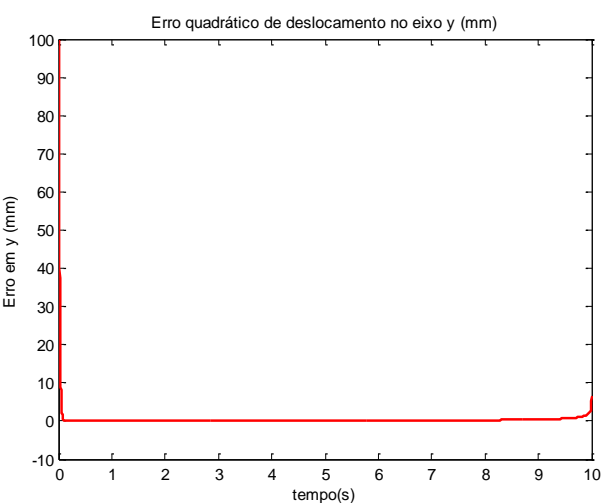

(b)

A Figura 9 ilustra que após o assentamento do sistema, os erros de posicionamento em $x$ e y, são de aproximadamente $0,3 \mathrm{~mm}$, ou seja, o erro do raio é de aproximadamente $e_{\text {raio }}=\sqrt{e_{x}^{2}+e_{y}^{2}}=\sqrt{0,3^{2}+0,3^{2}}=0,43 \mathrm{~mm}$. Portanto, para um raio de interpolação de $100 \mathrm{~mm}$ tem-se um erro de $0,43 \%$, indicando que mesmo na presença de distúrbio o sistema continua respondendo satisfatoriamente.

\section{CONCLUSÕES}

Modelou-se um sistema para controle de velocidade e posicionamento com quatro variáveis de estados discretizadas. Este tipo de abordagem é muito útil, pois permite que o modelo seja implementado diretamente em computadores digitais utilizando apenas algoritmos recursivos, o que torna a programação bastante simples. Os resultados obtidos nas simulações mostraram-se bastante coerentes e estão de acordo com o esperado, indicando que a modelagem abordada foi bem sucedida. A arquitetura de controle que foi proposta, também se mostrou bastante robusta, sendo imune inclusive a perturbações de torque da ordem de $20 \%$ do torque dinâmico (utilizado para escolha do motor).

Na sequência, a construção do protótipo da mesa $X-Y$ deverá ser retomado e testado para confrontação das simulações aqui descritas com resultados experimentais.

\section{REFERÊNCIAS}

BREAZ R. et al. Motion control of medium size CNC machine-tools-A hands-on approach. Conference Paper. July 2012.

GUETHI, Breno L. M. Estudo de cortadoras a laser open source e seus problemas mais comuns. Mecatrone, 2017.

https://doi.org/10.11606/issn.2526-8260.mecatrone.2017.134667

OGATA, K. Discrete Time Control Systems. 2nd edition. Prentice Hall, 1995.

PANDILOV, Z.; DUKOVSKI, V. Analytical calculation of the CNC machines servo drives position loop gain. Journal of Achievements in Materials and Manufacturing Engineering. Vol. 37 - Issue 2 - December 2009.

PANDILOV, Z.; DUKOVSKI, V. Analytical calculation of the CNC machines position loop gain. Journal of Achievements in Materials and Manufacturing Engineering. Vol. 23. Issue 1. July 2007. 
ROTHBART, A. HAROLD; BROWN JR, H. THOMAS. Mechanical Design Handbook: Measurement, analysis and control of dynamic systems. 2. ed. MacGraw-Hill, 2006. VU, H.; ESFANDIARI, R. Dynamic Systems Modeling and Analysis. Singapore: MacGraw-Hill International, 1998.

YOSHIKAWA, Mariana W.; Branco, Kalenin de M. Desenvolvimento de impressora 3D de coordenadas polares. Mecatrone, 2015.

https://doi.org/10.11606/issn.2526-8260.mecatrone.2015.114306

ZHAO, G. et al. Research on Nonlinear PID Position Controller of CNC System. Proceedings of the IEEE International Conference on Automation and Logistics. China, August 21, 2007.

ZHAO, G. et al. Research on Position Controller of CNC Machine. Second International Conference on Intelligent Computation Technology and Automation. China, 2009.

ZHOU XIN. The study of closed-loop position control system based on CNC processing. Advanced Materials Research. Switzerland, 2014.

\title{
Title: Development of a Didactic X-Y Table for Multidisciplinary Teaching in Mechatronics Engineering
}

\begin{abstract}
This paper describes a didactic system under development for multidisciplinary teaching in Mechatronics Engineering, involving Mechanical Design, Programming, Embedded Systems, Real-Time Systems, and Control. It is a Cartesian X-Y system driven by $D C$ servo motors and controlled by microcontrollers of high computational power, which allows the configuration by software of different experiments, network connection, and remote operation. This work focuses on detailed, step-by-step dynamic modeling of the system, which can later be worked on in more advanced disciplines. The modeling uses a hybrid approach to control speed and position, and the results of simulations are presented. Torque disturbances were also included, and the system proved to be robust.
\end{abstract}

Keywords: Multidisciplinary teaching. Mechatronics Engineering. Virtual instrumentation, Modeling and control. 